\title{
Introduction to the Special Issue on Advances in Cytokinesis Research
}

\author{
Issei Mabuchi \\ Division of Biology, Graduate School of Arts and Sciences,University of Tokyo, Komaba, Meguro-ku, Tokyo, \\ 153-8902, and Department of Cell Biology, National Institute for Basic Biology, Okazaki, Aichi 444-8585, \\ Japan
}

Animal cells and primitive eukaryotic cells such as fission yeast and cellular slime mold amoeba divide by the contraction of the contractile ring formed at the division plane of the cell. Recent studies have shown that a similar ring is formed during cytokinesis in the budding yeast Saccharomyces cerevisiae, one of the most primitive eukaryotes (see Bi, this issue). Our knowledge in this field has been accumulating quite rapidly in recent years. Therefore, it would be of interest and value to make a collection of short reviews by scientists in this field at this moment in order to understand what has been done and what has yet to be done. That is the reason why the publication of this special issue was undertaken.

The contractile ring is a belt of actin filament bundles that underlie the plasma membrane and encircle the cell at the division site. The actin filaments in the contractile ring are connected to the plasma membrane in an end-on fashion at their barbed end (Mabuchi et al., 1988), and arranged in an antiparallel manner which enables the filaments to slide over another provided that myosin filaments are present between them (Sanger and Sanger, 1980; Mabuchi et al., 1988). The contractile ring actually contains myosin II as a molecular motor that generates the contractile force of the contractile ring (Fujiwara and Pollard, 1976; Mabuchi and Okuno, 1977; see also Nagasaki et al., this issue). However, how myosin II is positioned in the structure of the contractile ring is not clear at present.

The contractile ring is a transiently formed structure that is fully formed typically during anaphase to telophase of mitosis, becomes smaller in volume during contraction, and finally disappears after completion of cytokinesis as elaborately studied with sea urchin eggs by Tom Schroeder in 1972. Thus, in spite of the fact that the contractile ring is composed of actin filaments and myosin II similar to muscles, the assembly properties of these proteins in nonmuscle cells during cytokinesis is quite dynamic as compared to those in myofibrils. Actin and myosin assemble during the

\footnotetext{
Division of Biology, Graduate School of Arts and Sciences,University of Tokyo, Komaba, Meguro-ku, Tokyo, 153-8902, and Department of Cell Biology, National Institute for Basic Biology, Okazaki, Aichi 444-8585, Japan.

Tel: +81-3-5454-6630, Fax: +81-3-5454-4318

E-mail: mabuchi@ims.u-tokyo.ac.jp
}

formation of the contractile ring, but how do these proteins assemble at the division site? Do these proteins form filaments at an early step, which then assemble together to form the contractile ring? Or do preexistent filaments of these proteins assemble together during this process? Our knowledge as to how these proteins behave during formation of the contractile ring has been limited. Contraction of the ring occurs in the next step, and, curiously enough, disassembly of actin filaments and myosin occurs during the contraction. Does the process of disintegration of the contractile ring during its contraction occur in reverse order to that of its formation? What is the mechanism of the transition from the assembly phase to the disassembly phase and how is the disassembly phase coupled to contraction? There has been no answer to these questions.

It has been considered that various actin-modulating proteins are responsible for the dynamic property of actin during cytokinesis. Actually, a number of actin-modulating proteins have been found to be necessary for cytokinesis. Recent genetical studies using fission yeast (Balasubramanian et al., 1993; Wu et al., 2001), Dictyostelium amoeba (see Weber, this issue), and Drosophila melanogaster embryos (see Giansanti et al., this issue) have greatly contributed to the discovery of these proteins. Some of these proteins are considered to be necessary in the formation of the contractile ring. However, the steps in the process of cytokinesis where these proteins are really functioning have yet to be known. This is an important problem that must be clarified in order to understand how actin filaments are accumulated at the division site and organized into the contractile ring, and how the contractile ring actin filaments are disintegrated during contraction of the ring. It is also important for understanding how these processes are controlled by upstream signaling pathways since the function of these proteins must be controlled by such pathways.

Being different from actin assembly, the assembly of myosin in the nonmuscle cells of higher animals may be regulated by the phosphorylation of its regulatory light chain. The myosin regulatory light chain has been shown to be phosphorylated during mitosis, with these phosphorylated light chains accumulating in the division plane (Satterwhite et al., 1992; see Matsumura et al., this issue; see 
Iwasaki et al., this issue). This phosphorylation is proposed to be under the control of the Rho-signaling pathway (see below); however, the entire picture has yet to be uncovered. On the other hand, it has been shown that the phosphorylation of the regulatory light chain is not necessary for the function of myosin in cell division in Dictyostelium amoeba (Ostrow et al., 1994). Furthermore, it is reported that the initial assembly of myosin at the division site requires neither its head domain nor presence of F-actin in lower eukaryotes (Yumura and Uyeda, 1997; Zang and Spudich, 1998; Naqvi et al., 1999; see Noguchi et al., this issue). This suggests that there may be other mechanisms for the assembly of myosin at the division site. It is thus necessary to determine whether a mechanism of myosin assembly exists at the division site, which is commonly used among various eukaryotic cells.

The timing and the position of formation of the contractile ring must be precisely controlled in order to properly distribute chromosomes and cytoplasmic components among the daughter cells. It has been proposed that the position of the division plane in animal cells, where the contractile ring assembles, is determined by astral or central microtubules of the mitotic apparatus (Rappaport, 1996; see Hamaguchi et al., this issue; Wang, this issue; Bowerman, this issue; Giansanti et al., this issue). Thus a signal for cleavage may be transmitted from the microtubules to the cell cortex, where it is able to assemble actin filaments and myosin, or establish the conditions for accumulation of these proteins. In the case of fission yeast in which the mitotic spindle is formed inside of the nucleus, such signaling is considered to be executed by the nucleus (see Chang, this issue).

Elucidation of the cleavage signal has been one of the most exciting themes in cytokinesis research. One breakthrough is the discovery that the small G-protein Rho is involved in the cleavage signaling (Kishi et al., 1993; Mabuchi et al., 1993). Findings that regulators of Rho GTPase activity are also involved in cleavage have contributed to establishment of the involvement of the Rho-signaling pathway (see O'Keefe et al., this issue; Kitamura et al., this issue; Adachi, this issue). The Rho-pathway may be able to regulate both the polymerization of actin through diaphanous/formin family proteins (Watanabe et al., 1996; Chang et al., 1997; see Bowerman, this issue) and the phosphorylation of myosin regulatory light chain through Rho-activated kinases and myosin phosphatase (see Matsumura et al., this issue).

The other signaling molecules which may function in cytokinesis are the so-called chromosomal passenger proteins, which have been known to change their localization during cell division from chromosomes to the midbody. Among these proteins, INCENP, Aurora kinase, and survivin may form a complex and are involved in both mitosis and cytokinesis (see Adams et al., 2001; Wang, this issue; Terada, this issue; Bowerman, this issue), and while it has been known that these molecules are involved in a late step of cytokinesis, it raises the possibility that they somehow link these two events of cell division.

Many of the findings described above have been obtained quite recently. The detailed stories of these findings are to be found in the articles in this issue. Although a lot of riddles remain as to the mechanism of cytokinesis as mentioned above, we may expect them be solved to a large extent in the near future.

\section{References}

Adams, R.R., Carmena, M., and Earnshaw, W.C. 2001. Chromosomal passengers and the (aurora) ABCs of mitosis. Trends in Cell Biol., 11: 4954

Balasubramanian, M.K., Hirani, B.R., Burke, J.D., and Gould, K.L. 1994. The Schizosaccharomyces pombe $c d c 3^{+}$gene encodes a profilin essential for cytokinesis. J. Cell Biol., 125: 1289-1301.

Chang, F., Drubin, D., and Nurse, P. 1997. cdc12p, a protein for cytokinesis in fission yeast, is a component of the cell division ring and interacts with profilin. J. Cell Biol., 137: 169-182.

Fujiwara, K., and Pollard, T.D. 1976. Fluorescent antibody localization of myosin in the cytoplasm, cleavage furrow and mitotic spindle of human cells. J. Cell Biol., 71: 848-875.

Jantsch-Plunger, V., Gonczy, P., Romano, A., Schnabel, H., Hamill, D., Schnabel, R., Hyman, A.A., and Glotzer, M. 2000. CYK-4: A Rho family GTPase activating protein (GAP) required for central spindle formation and cytokinesis. J. Cell Biol., 149: 1391-1404.

Kishi, K., Sasaki, T., Kuroda, S., Itoh, T., and Takai, Y. 1993. Regulation of cytoplasmic division of Xenopus embryo by rho p21 and its inhibitory GDP/GTP exchange protein (rho GDI). J. Cell Biol., 120: 1187-1195.

Mabuchi, I. and Okuno, M. 1977. The effect of myosin antibody on the division of starfish blastomeres. J. Cell Biol., 74: 251-263.

Mabuchi, I., Tsukita, S., Tsukita, S., and Sawai, T. 1988. Cleavage furrow isolated from newt eggs: contraction, organization of the actin filaments and protein components of the furrow. Proc. Nat. Acad. Sci. USA, 85: 5966-5970.

Mabuchi, I., Hamaguchi, Y., Fujimoto, H., Morii, N., Mishima, M., and Narumiya, S. 1993. A rho-like protein is involved in the organisation of the contractile ring in dividing sand dollar eggs. Zygote, 1: 325-331.

Naqvi, N.I., Eng, K., Gould, K.L., and Balasubramanian, M.K. 1999. Evidence for F-actin-dependent and -independent mechanisms involved in assembly and stability of the medial actomyosin ring in fission yeast. EMBO J., 18: 854-862.

Ostrow, B.D., Chen, P., and Chisholm, R.L. 1994. Expression of a myosin regulatory light chain phosphorylation site mutant complements the cytokinesis and developmental defects of Dictyostelium RMLC null cells. J. Cell Biol., 127: 1945-1955.

Rappaport, R. 1996. Cytokinesis in animal cells. Cambridge University Press, Cambridge, UK.

Sanger, J.M. and Sanger, J.W. 1980. Banding and polarity of actin filaments in interphase and cleaving cells. J. Cell Biol., 86: 568-575.

Satterwhite, L.L., Lohka, M.J., Wilson, K.L., Scherson, T.Y., Cisek, L.J., Corden, J.L., and Pollard, T.D. 1992. Phosphorylation of myosin-II regulatory light chain by cyclin-p34 ${ }^{\mathrm{cdc} 2}$ : a mechanism for the timing of cytokinesis. J. Cell Biol., 118: 595-605.

Schroeder, T.E. 1972. The contractile ring. II. Determining its brief existence, volumetric changes, and vital role in cleaving Arbacia eggs. J. Cell Biol., 53: 419-434.

Watanabe, N., Madaule, P., Reid, T., Ishizaki, T., Watanabe, G., Kakizuka, A., Saito, Y., Nakano, K., Jockush, B.M., and Narumiya, S. 1997. 
p140mDia, a mammalian homolog of Drosophila diaphanous, is a target protein for Rho small GTPase and is a ligand for profilin. EMBO J., 16: 3044-3056.

Wu, J., Bähler, J., and Pringle, J.R. 2001. Role of a fimbrin and an $\alpha$-actinin-like protein in fission yeast cell polarization and cytokinesis. Mol. Biol. Cell, 12: 1061-1077.
Yumura, S. and Uyeda, T.Q.P. 1977. Transport of myosin II to the equatorial region without its own motor activity in mitotic Dictyostelium cells. Mol. Biol. Cell, 8: 2617-2629.

Zang, J.H. and Spudich, J.A. 1998. Myosin II localization during cytokinesis occurs by a mechanism that does not require its motor domain. Proc. Nat. Acad. Sci. USA, 95: 13652-13657. 